\title{
Study on Fabrication of Antibacterial Low Molecular Weight Nanochitosan Using Sodium Tripolyphosphate and Hydrogen Peroxide
}

\author{
Tuan Anh Nguyen (D) and Thi Huong Nguyen \\ Faculty of Chemical Technology, Hanoi University of Industry (HaUI), No. 298 Cau Dien, District Bac Tu Liem, Hanoi, Vietnam \\ Correspondence should be addressed to Tuan Anh Nguyen; anhnt@haui.edu.vn
}

Received 28 August 2021; Accepted 21 December 2021; Published 17 January 2022

Academic Editor: Brajesh Kumar

Copyright (C) 2022 Tuan Anh Nguyen and Thi Huong Nguyen. This is an open access article distributed under the Creative Commons Attribution License, which permits unrestricted use, distribution, and reproduction in any medium, provided the original work is properly cited.

\begin{abstract}
In the study, chitosan was decomposed into low molecular weight chitosan by using different $\mathrm{H}_{2} \mathrm{O}_{2}$ concentrations for chain termination, and then chitosan was prepared with different concentrations of tripolyphosphate cations (TPP). We have obtained the following result: the formation of chitosan depends on the concentration of TPP; TPP at low or high concentrations does not react with chitosan to form small chitosan molecules. Properly structured chitosan is only obtained when the mass ratio of chitosan/TPP is $6: 3$. At this rate, when the mass ratio of chitosan/TPP is approximately $6: 3$, there the formed nanochitosan particles have good antibacterial ability against strains of E. coli, M. catarrhalis, and S. aureus. In this work, initially, a successful preparation of a suspension between nasal spray and small chitosan suspension was found at manufacturing ratios: $5 \mathrm{ml}$ nasal spray and $5 \mathrm{ml}$ manufactured chitosan suspension at concentrations of $1 \mathrm{mg} / \mathrm{ml}, 3 \mathrm{mg} / \mathrm{ml}$, and $5 \mathrm{mg} / \mathrm{ml}$. It proves that the chain termination process by using $\mathrm{H}_{2} \mathrm{O}_{2}$ and the creation cross-linking when adding TPP are successful to a certain extent.
\end{abstract}

\section{Introduction}

Nanochitosan is a natural material with excellent physicochemical properties that makes it an environmentally friendly material, whose biological activity is not harmful to humans. It is often used as a pharmaceutical carrier and also has antibacterial properties. Nanochitosan has been used to improve antibacterial effects in the manufacture of drug suspensions [1]. The potential to use chitosan in various fields such as antibacterial, antifungal, antiviral, antacid, nontoxic, biodegradable, and biocompatible with animal and plant tissues, also such as membrane formation, has attracted strong interest for developments in the field of biomedicine [2]. Nanochitosan is prepared in many different ways, such as creating covalent and ionic bonds. First, the ionic cross-linking method is used to prepare nanochitosan particles. By adding tripolyphosphate cations (TPP) to the chitosan solution, a cross-linking between reaction molecules between the free amino groups of chitosan and TPP cations was created, creating a chitosan gel granule. A number of other studies have conducted nanochitosan synthesis from low molecular weight chitosan by using $\mathrm{H}_{2} \mathrm{O}_{2}$ as an oxidant to cut molecules [3]. Pant et al. studied materials based on PU (polyurethane) nanofibers with salicylic acid and silver nanoparticles. Using electrospinning method, nanomaterials with high antibacterial ability and good mechanical properties have been successfully fabricated $[4,5]$. Chitosan nanoparticles are prepared from low and medium molecular weight chitosan. The results show that CSNPs can be widely applied as antimicrobial agents in medicine and food science for their antibacterial activity and high biocompatibility [6]. O'Callaghan et al. used ionic gelation to generate low and medium molecular weight chitosan nanoparticles. Nanoparticles ( $1 \%$ acetic acid) have been shown to have high antibacterial activity [7]. From the studies demonstrated, the selection of hydrolyzed chitosan remains a challenge. The aim is to obtain oligomers with specific sizes. The hydrolysis products are chitosan and 
chitooligosaccharides of medium and low molecular weight, with bioactive properties. New technologies and research focusing on the use of chitosan hydrolysates have been continuously introduced in the scientific literature [8]. With antibacterial ability, nanochitosan is also studied in the application of anthocyanin drugs [9]. In addition, using chitosan-based hydrogel as the delivery platform for peptides (Ps) and hydrogen peroxide (HB) may provide a more effective therapy in wound healing [10]. Chitosan (CS) is a biopolymer with the characteristics of high biocompatibility and biodegradability, so it is very convenient for biomedical applications. Safarzadeh et al. fabricated chitosan nanoparticles using a unique combination of $\mathrm{H}_{2} \mathrm{O}_{2}$-assisted decomposition, ultrasound, and ionic gelation [11].

Although there are many researches on nanochitosan manufacturing, there are currently no researches on nanochitosan fabrication from chitosan molecule with low molecular weight and assessing antibacterial ability. In this work, we have made nanochitosan from low molecular weight chitosan and it has been initially used in the preparation of a suspension with a good antibacterial nasal spray.

\section{Materials and Methods}

2.1. Materials. The following materials were used: chitosan (CS) (deacetylation >75-85\%, $1.61 \times 105 \mathrm{Da}$ ) (manufactured by Aldrich); sodium tripolyphosphate, potassium dichromate, and AR type acetic acid were used without further purification; $\mathrm{H}_{2} \mathrm{O}_{2}, \mathrm{MgCl}$ (Osaka, Japan); nasal sprays from iridescent flowers (Ageratum conyzoides L.) (Institute of Medicinal Materials, Vietnam); $1 \%$ acetic acid $\left(\mathrm{CH}_{3} \mathrm{COOH}\right)$ is made from $99.5 \%$ acetic acid (China); ethanol (China).

2.2. Specimen Preparation. First 2 grams of chitosan were dissolved in $100 \mathrm{ml}$ of $0.1 \mathrm{M} \mathrm{HCl}$ and stirred for 45 minutes. Subsequently, $\mathrm{H}_{2} \mathrm{O}_{2}$ was added to one of the four concentrations $(4 \% ; 6 \% ; 8 \%$; and $10 \%)$. The mixture was heated and stirred at $65 \mathrm{C}$ for $2 \mathrm{~h}$ and then vacuum filtered. The residue is neutralized with distilled water, baked, and weighed. Ethanol was added to the lower solution, which was left for 24 hours to precipitate, after which it was filtered, dried, and weighed. These water-soluble low molecular weight chitosans are designated CS4 CS6, CS8, and CS10. The viscosity of the suspension was measured at $25^{\circ} \mathrm{C}$ using the Ostwald viscometer (Germany). Next, $0.5 \mathrm{~g}$ of chitosan (CS4, CS6, CS8, and CS10) was dissolved in $1000 \mathrm{ml}$ of $2 \%$ acetic acid and stirred for 25 minutes. Then, $100 \mathrm{ml}$ of each solution was added to $40 \mathrm{ml}$ of $\operatorname{TPP}(0.2,0.3,0.4$, and $0.5 \mathrm{~g} / \mathrm{L})$, stirring for 2 hours at ambient temperature for 30 minutes to obtain the solution. The solution is clear white and then centrifuged at a high speed of $5000 \mathrm{rpm}$. The chitosan after fabrication has low molecular weight (LWCS low molecular weight chitosan) $[11,12]$.

\subsection{Characterization and Testing}

2.3.1. Determination of Antibacterial Ability (Figure 1). Method: testing the bacteriostatic activity by diffusing agar plate. The bacteriostatic activity test method is the method of
Hadacek et al. (2000), measured at the Institute of Biotechnology, Vietnam Academy of Science and Technology.

Strains tested for bacteria: Escherichia coli ATCC 25922; Staphylococcus aureus ATCC 12222; and Moraxella catarrhalis ATCC 49143.

\section{Results and Discussion}

3.1. Manufacturing Low Molecular Weight Chitosan by Using Oxidizing Agent $\mathrm{H}_{2} \mathrm{O}_{2}$. During the experiment, the reaction was kept constant under reaction time of 2 hours and the reaction temperature was $60^{\circ} \mathrm{C}$; we only consider the effect of the amount of $\mathrm{H}_{2} \mathrm{O}_{2}$ on the resulting product. Because $\mathrm{H}_{2} \mathrm{O}_{2}$ is an oxidizing agent that reacts directly with chitosan, the low molecular weight chitosan obtained will be greatly affected by the content of $\mathrm{H}_{2} \mathrm{O}_{2}$.

From Figure 2, after treatment with $\mathrm{H}_{2} \mathrm{O}_{2}$, the viscosity of the solution after heating decreases and continues to decrease with increasing the content of $\mathrm{H}_{2} \mathrm{O}_{2}$. This demonstrates that the mass of the original chitosan molecule has been reduced. There was a cessation reaction of chitosan initially with $\mathrm{H}_{2} \mathrm{O}_{2}$, in which $\mathrm{H}_{2} \mathrm{O}_{2}$ acts as an oxidizing agent that breaks down the original chitosan molecules into smaller molecules.

The amount of product obtained more or less depends on the amount of $\mathrm{H}_{2} \mathrm{O}_{2}$. With the content of $\mathrm{H}_{2} \mathrm{O}_{2}$ being $15 \%$ compared to the initial amount of chitosan, after adding ethanol to precipitate, the amount of the resulting product is very small, leaving only the solution in the cup slightly opaque.

When increasing the amount of $\mathrm{H}_{2} \mathrm{O}_{2}$ to $20 \%$, after adding ethanol, the beaker appears to precipitate as a suspension in the solution, making the solution in the cup white opaque. As the amount of $\mathrm{H}_{2} \mathrm{O}_{2}$ increases, the molecular cutting oxidation occurs stronger, the amount of product is greater, and the solution obtained in the cup is suspension with small suspended particles evenly distributed in the liquid. When the amount of $\mathrm{H}_{2} \mathrm{O}_{2}$ reaches $50 \%$, the amount of product obtained is the most.

After 3 days of settling, small particles settle to form a white opaque layer at the bottom of the cup. Washing with distilled water several times will result in an opalescent precipitate in the form of spongy cotton, deposited at the bottom of the beaker. The solution in the beaker is transparent and has a neutral medium.

After conducting lyophilization, a low molecular weight chitosan sample produces a spongy opalescent solid (see Figure 3).

\subsubsection{Infrared Spectrum of Low Molecular Weight Chitosan.} Figures 4 and 5 are the infrared spectra of the original chitosan sample and the low molecular weight chitosan sample prepared by infrared spectrometer at the Institute of Chemistry, Vietnam Academy of Science and Technology.

Both spectra have characteristic peaks for the appearance of the $\mathrm{OH}$ group. For the original chitosan sample, the absorbance peak is at 3423. For the low molecular weight chitosan sample, the absorbance peak is at 3442 . 


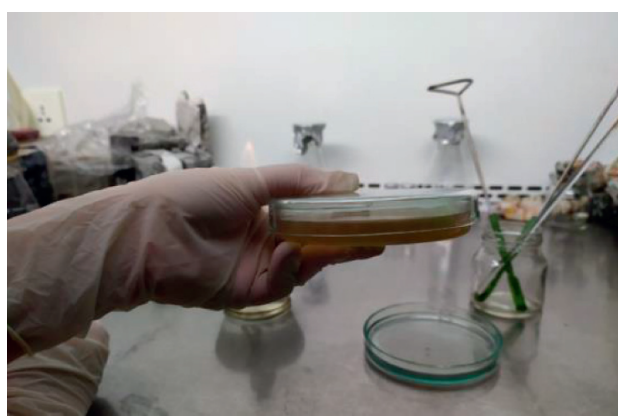

Prepare the plates

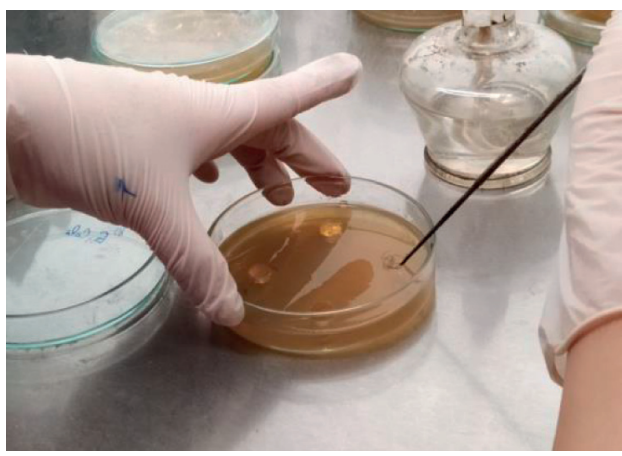

Drill wells and small samples

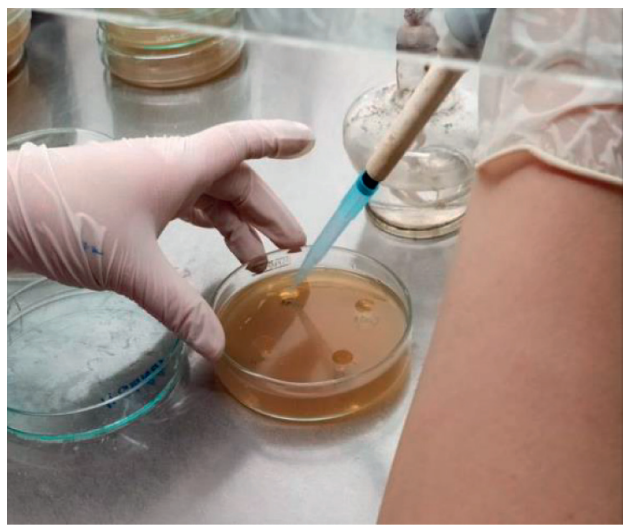

Small sample into the hole and incubated

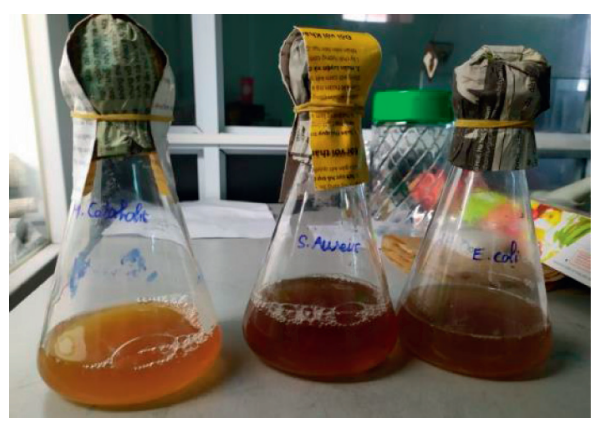

Preparing for testing strains of bacteria

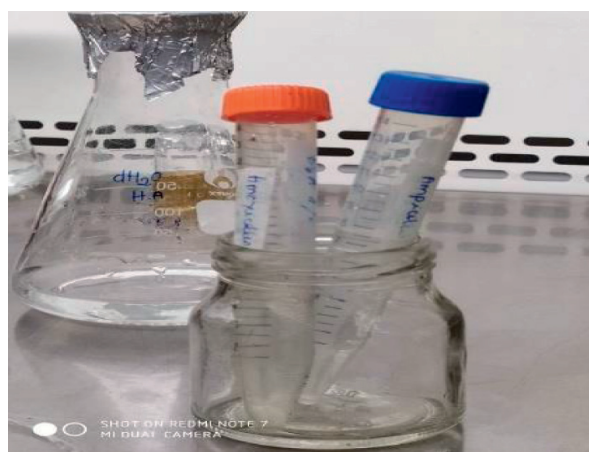

Control sample

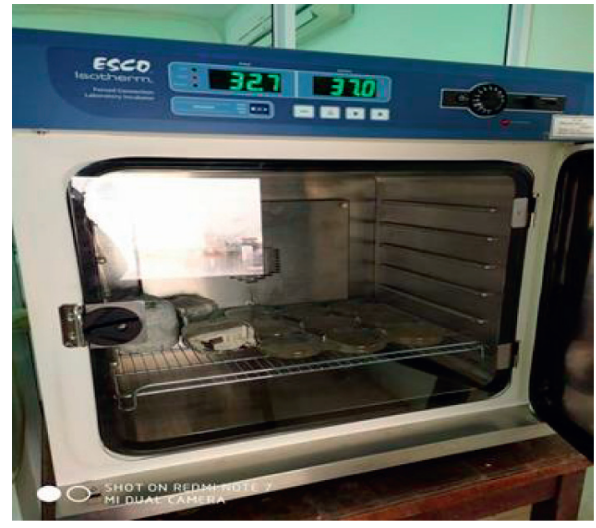

e antibacterial ability.
Peak 2880 in the spectrum of the original chitosan sample and peak 2918 in the low molecular weight chitosan sample characterized the valence vibrations of the $\mathrm{CH}$ group.

On the infrared spectrum of the original chitosan sample, the absorption band characteristic for the valence vibrations of $\mathrm{NH}_{2}$ group is shown at peak 1652. For low molecular weight chitosan samples, the peak corresponding to the $\mathrm{NH}_{2}$ group is valued at 1662 .

In general, for modulated samples, the characteristic peaks for oscillation of functional groups are not much different from the infrared spectrum.

3.2. Viscosity Survey. After treatment with $\mathrm{H}_{2} \mathrm{O}_{2}$, the molecular weight and viscosity of chitosan decrease and continue to decrease with increasing of the content of $\mathrm{H}_{2} \mathrm{O}_{2}$ (Table 1). This is due to the degradation of the chitosan molecular chain by $\mathrm{H}_{2} \mathrm{O}_{2}$, which oxidizes the $\mathrm{OH}$ and $\mathrm{NH}_{2}$ groups to the $\mathrm{COOH}$ group. Therefore, the $\mathrm{N}$ content of chitosan $(L)$ decreases as the volume of $\mathrm{H}_{2} \mathrm{O}_{2}$ increases, while $\mathrm{O}$ content increases (Table 1).

The antibacterial result of low molecular weight chitosan is shown in Figure 6.

From Figure 6, the antibacterial ability of low molecular weight chitosan was tested with 3 strains of bacteria, namely, S. aureus, E. coli, and M. catarrhalis. Đc (+) well is dripped with a special antibiotic containing low molecular weight chitosan. Đc (-) well contains distilled water or physiological saline. If the chitosan suspension is antibacterial, a zone of inhibition will appear around Đc $(+)$ well.

The zone of inhibition is an area around the cavity where the bacteria have not grown enough to be visible. The zone of inhibition can be easily identified because the bacterial suspension is turbid according to the standard. The ring of no bacterial growth will be clearer. 


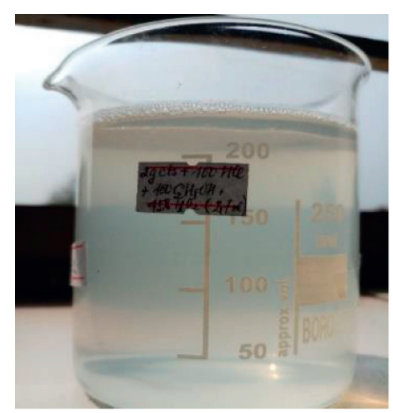

(CS4)

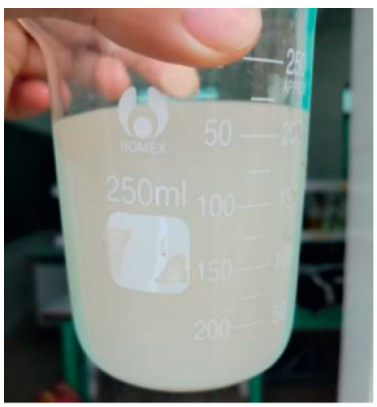

(CS6)

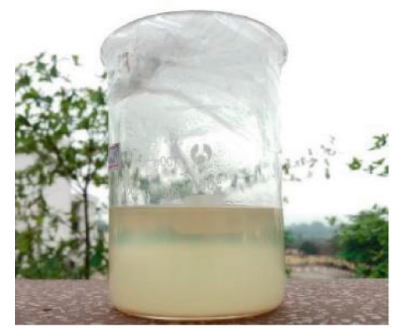

(CS8)
FIgUre 2: Oxidation of CS with $\mathrm{H}_{2} \mathrm{O}_{2}$.

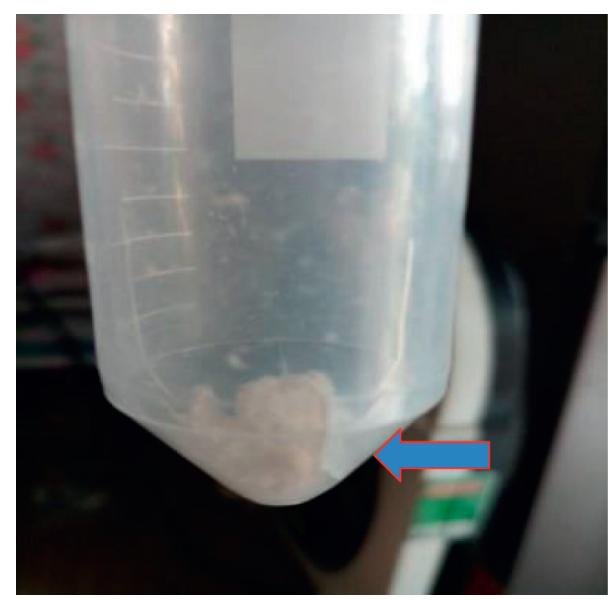

FIGURE 3: Low molecular chitosan after conducting lyophilization.

The above results show that

Đc (-) well containing distilled water or physiological saline produces no zone of inhibition for all 3 samples, showing no antibacterial ability

Đc (+) well produces the zone of inhibition for samples $3-a$ and $3-b$, showing that the chitosan suspension has antibacterial ability against $S$. aureus and E. coli bacteria

\subsection{Manufacturing Chitosan of Small Size by Using Sodium} Tripolyphosphate (TPP). After completely dissolving chitosan in acetic acid solution, adjust the $\mathrm{pH}$ of chitosan solution with $\mathrm{NaOH} 5 \mathrm{~N}$ solution. Then, proceed slowly to drip the sodium tripolyphosphate (TPP) solution at a concentration of $1 \mathrm{mg} / \mathrm{ml}$ (diluted in distilled water) into the previous solution.
The chitosan particles are formed, which will appear as suspended in solution and the solution is centrifuged by a high-speed cold centrifuge, with a velocity of $20.000 \mathrm{rpm}$ for 30 minutes. After centrifuging, remove the aqueous layer above, obtain the solid layer underneath, and wash several times with distilled water. NCTS particles were vacuum freezing dried by Labconco FreeZone freeze-dried machine for FE-SEM, IR, and XRD measurement.

The CS/TPP ratio is a very important factor influencing the size of chitosan nanoparticles. In the world, there have been many studies on them and they produced many results. In particular, according to Gan's group, the more the CS/ TPP ratio increases, the more the particle size increases, the modulated particle size ranges from 100 to $250 \mathrm{~nm}$. While Bing Hu provides the opposite result, the more the CTS/TPP ratio increases, the smaller the particle size.

When gradually adding TPP solution to chitosan solution, the phenomenon is that the solution is getting more and more limpid while the CTS/TPP ratio increases. Besides, it can be seen that the chitosan particles have formed, as shown in Figure 7. After centrifuging, remove the supernatant layer, obtain the solid layer underneath, and wash several times with distilled water. Then, put the clean solution containing solids into the vial.

After a few minutes, it is found that the solutions M1, M2, M3, and M4 are not separated and have a more opaque color than other solutions, showing that these particles are small in size and difficult to settle. In the remaining proportions, it is evident that the particles begin to accumulate at the bottom of the vial; due to the large size and mass of the particle, it is easier to settle.

The results of the two infrared spectra show that there is a difference between the absorption peaks of CTS and NCTS.

On the IR spectrum of chitosan (Figure 8), the absorption peak is at $3423.4 \mathrm{~cm}^{-1}$ with a well-rounded shape showing the absorption wave bands typical for the valence band of -OH group, while for the spectrum of nanochitosan (Figure 8), the absorption peak of this group is at $3420.43 \mathrm{~cm}^{-1}$.

From the results in Table 2, the absorption peaks that characterize the asymmetric valence fluctuations and symmetry of the $-\mathrm{CH}$ group of chitosan are at $2920.87 \mathrm{~cm}^{-1}$ and $2880.61 \mathrm{~cm}^{-1}$. For nanochitosan, this peak has a value of $2920.43 \mathrm{~cm}^{-1}$.

Fluctuation of the $-\mathrm{NH}_{2}$ group of chitosan is shown at the absorption peak of $1652.21 \mathrm{~cm}^{-1}$. In the chitosan nano spectrum, an additional peak of $1553.4 \mathrm{~cm}^{-1}$ appeared due to the association between ammonium and phosphoric groups.

The absorption peak of $1247.57 \mathrm{~cm}^{-1}$ is characteristic for the oscillation of the $-\mathrm{P}=\mathrm{O}$ group; this oscillation only appears in nanochitosan.

We conclude that the ammonium group of CTS has horizontal bond with TPP to create CTS-TPP nano product. The absorption intensity through optical transmittance of CTS is stronger and clearer than that of NCTS, showing that CTS absorbs radiation more strongly than NCTS.

In the world, there have been many scientific studies on this subject such as that by Sreekumar et al. [12] and when compared with the results of the study, it can be determined 


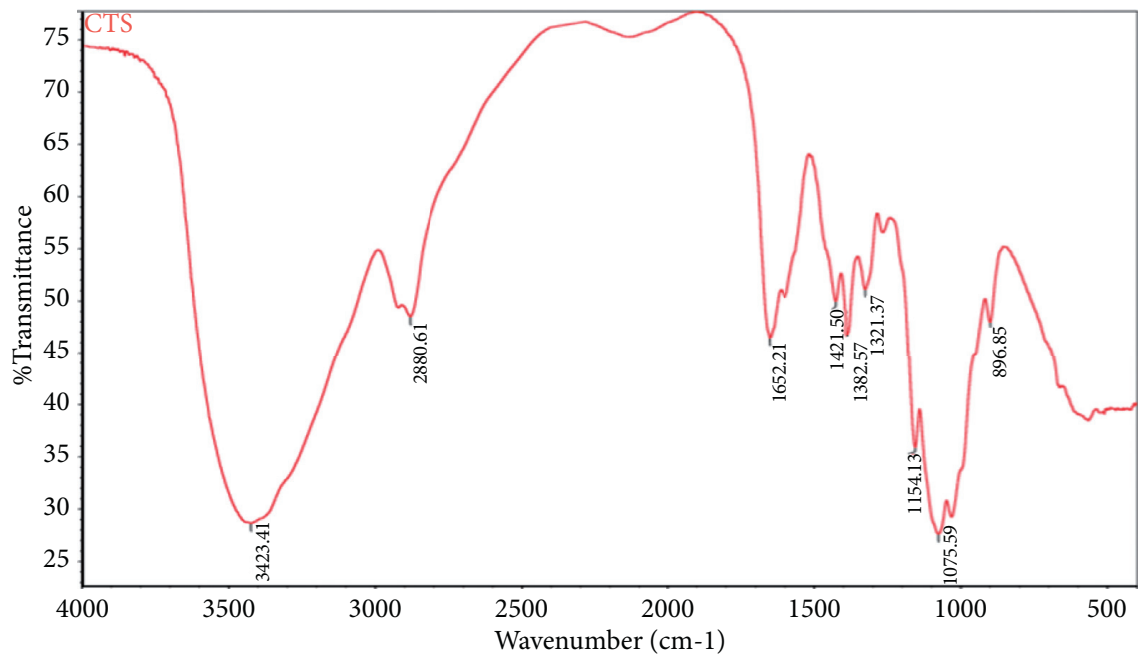

FIGURE 4: Infrared spectrum of the original chitosan sample.

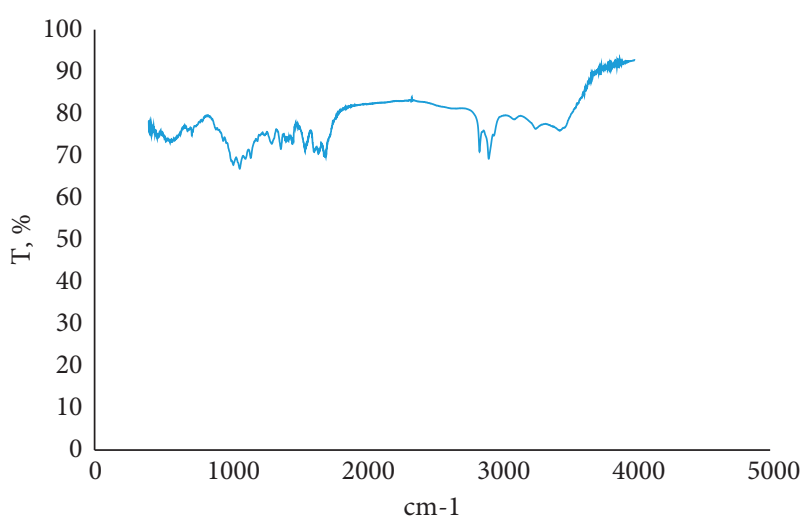

FIGURE 5: Infrared spectrum of low molecular weight chitosan was prepared with $6 \% \mathrm{H}_{2} \mathrm{O}_{2}$.

TABLE. 1: Molecular weight, viscosity, and elemental analysis of manufactured chitosan.

\begin{tabular}{lcccc}
\hline No. & Sample & $\mathrm{H}_{2} \mathrm{O}_{2}(\mathrm{ml})$ & $\mu(\mathrm{ml} / \mathrm{g})$ & $\mathrm{Mv}$ \\
\hline 1 & $\mathrm{CS} 4$ & 4 & 301.257 & 242.450 \\
$\mathbf{2}$ & $\mathrm{CS6}$ & $\mathbf{6}$ & $\mathbf{1 6 7 . 4 6 5}$ & $\mathbf{1 2 7 . 3 0 1}$ \\
3 & $\mathrm{CS} 8$ & 8 & 69.750 & 81.245 \\
4 & $\mathrm{CS} 10$ & 10 & 21.090 & 17.365 \\
\hline
\end{tabular}

Bold signifies that sample CS6 gives results for viscosity and molecular weight which are more suitable than other samples [1].

that NCTS is manufactured as a clean product and according to the requirements and criteria of NCTS.

3.3.1. Structural Morphology of Fabricated Chitosan. The morphological structure of the initial chitosan and chitosan after cross-linking after being cross-linked with TPP, observed through FE-SEM image, is shown in Figures 9 and 10. Figure 8 shows that the morphological structure of chitosan initially has the form of a polymer layer structure with large dimensions. By the method of creating cross-linking with TPP, the resulting chitosan is smaller in size (Figure 9), which ranges in size from $35 \mathrm{~nm}$ to $100 \mathrm{~nm}$ (average size is $67.50 \mathrm{~nm})$.

The nanometer size and uniform distribution are great potential for absorption for the treatment of disease playing an effective drug transmission through route of administration such as oral, blood, and especially inhalation.

The method of creating gel ion with TPP cross-linking (see Figure 11) agent is one of the simple, effective, nontoxic, and beneficially economic methods; hence, it will be selected for the following studies.

3.3.2. X-Ray Diffraction of Fabricated Chitosan. The degree of crystallinity of chitosan initially and chitosan after conducting cross-linking with TPP was assessed by X-ray diffraction diagram as shown in Figures 12 and 13.

The degree of crystallinity of the original chitosan material was assessed through their X-ray diffraction diagram. The $\mathrm{X}$-ray diffraction diagram is measured in the range of $2 \theta$ from 40 to 700 . The X-ray diffraction diagram of chitosan has two strong points at $2 \theta$ which are 100 and 200 , consistent with the diffraction diagram of chitosan published in the previous studies, showing the high degree of crystallinity chitosan raw material.

Figure 13 shows that no tip was found on the XRD diagram of chitosan-TPP after manufacturing. They show that the original structure of chitosan was destroyed after creating a cross-link with the TPP; this research result is consistent with the published results [13].

\subsection{The Antibacterial Ability of the Suspension of Chitosan} and Nasal Sprays from Ageratum conyzoides L. Firstly, testing DC (+) and DC (-) of the test disks, both have met the requirements to continue reading the results of the test samples (see Figures 14 and 15).

In particular, the sample 01 is the original Agerhinin drug and the sample 02 is the chitosan. From Figure 16, we see that the sample 01 has no antibacterial ability; there is no zone of inhibition for all 3 strains of bacteria tested. Hence, 


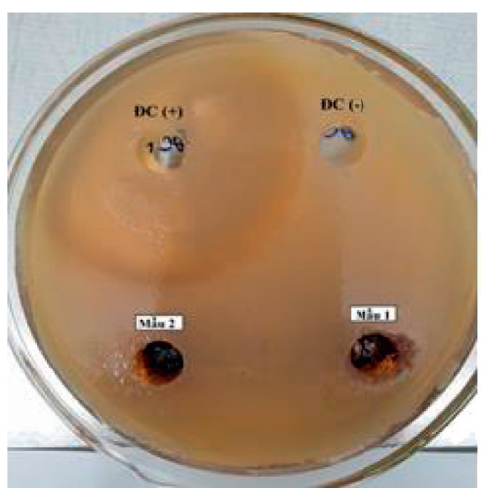

(a)

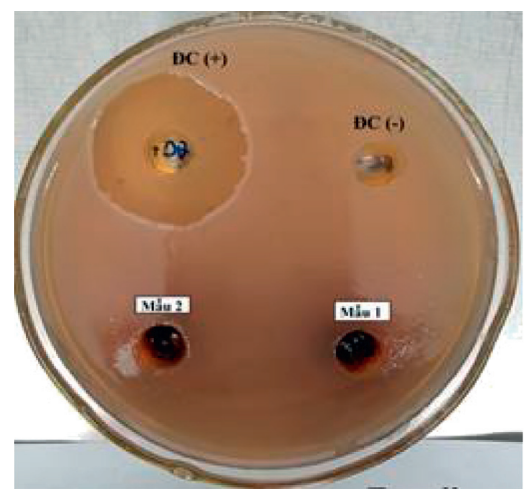

(b)

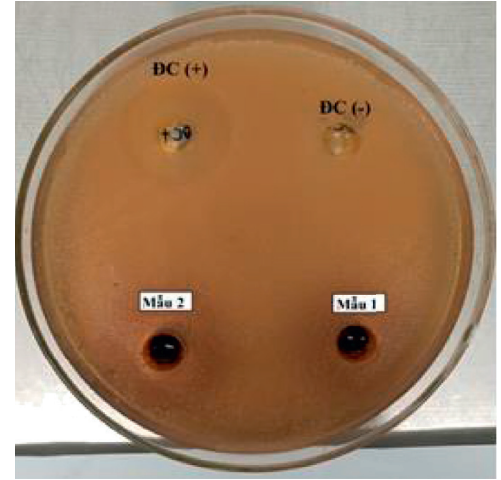

(c)

Figure 6: Antimicrobial results: (a) specimens with S. aureus; (b) specimen with E. coli; (c) specimen with M. catarrhalis.

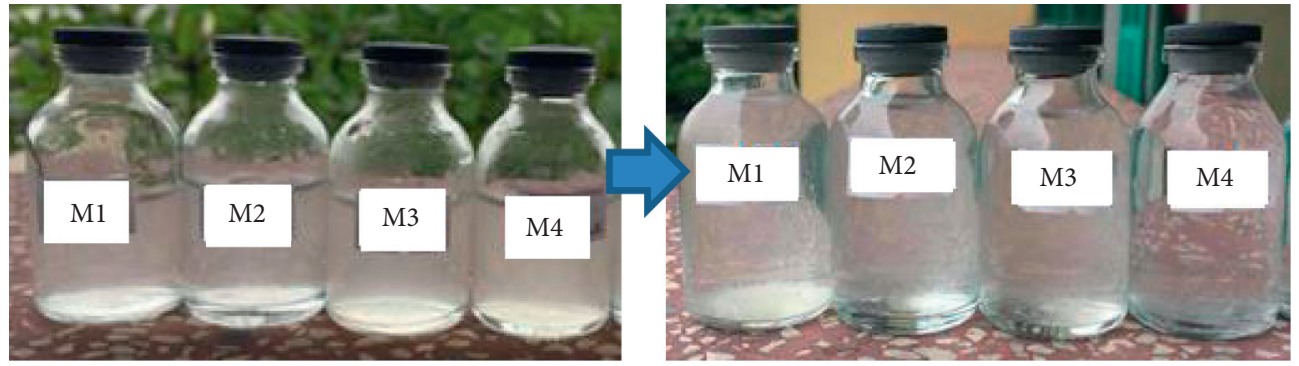

(a)

(b)

FIGURE 7: Chitosan solution at different CTS/TPP ratios before filtration with distilled water (a) and nanochitosan solution at different CTS/ TPP ratios after filtration with distilled water (b).

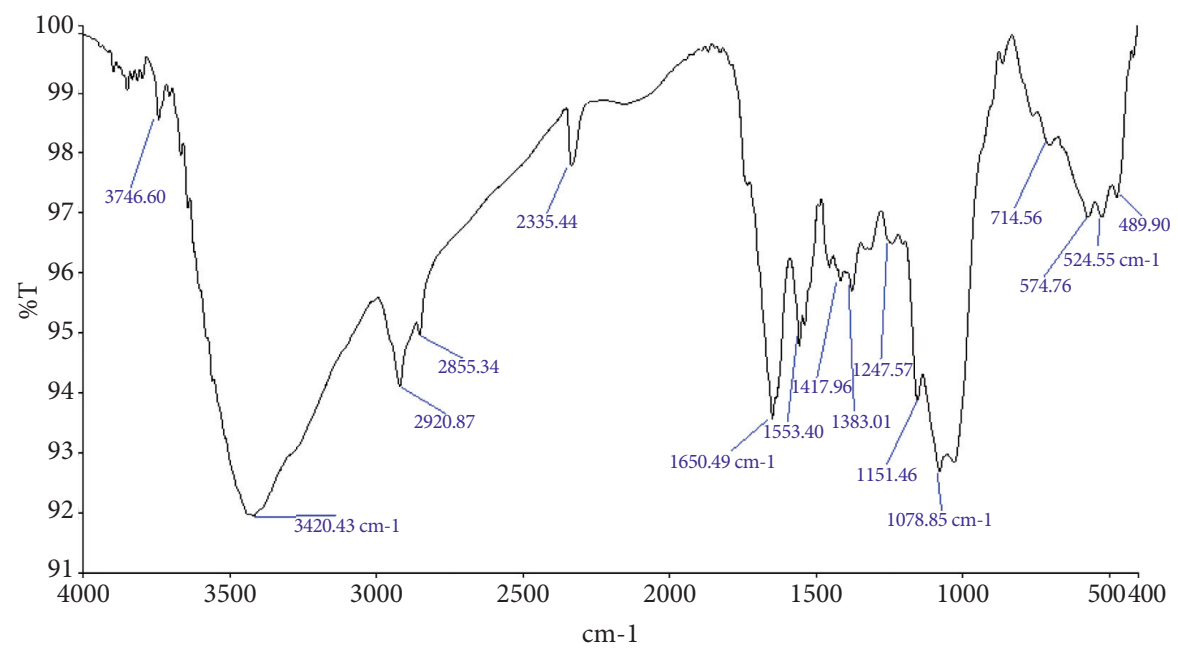

FIGURE 8: FT-IR spectrum of chitosan/TPP at manufacturing ratio 6/3.

the original Agerhinin drug solution has no antibacterial ability.

The sample 02 does not appear in the zone of inhibition for strains of E. coli and S. aureus; it is not resistant to these two types of bacteria. For M. catarrhalis bacteria, the sample 02 appears in the zone of inhibition but its size is only $1 \mathrm{~mm}$. Therefore, the sample 02 only showed signs of low antibacterial.
Thus, when nanochitosan was added to the drug solution, the drug sample did not have antibacterial ability initially, then appeared antibacterial signs, thereby confirming the antibacterial properties of Chitosan when combined with the drug.

From the results of the antibacterial evaluation, Figure 17 , it can be seen that the zone of inhibition appears clearly for all three bacterial strains, showing that the test 
TABLE. 2: The main absorption peaks $\left(\mathrm{cm}^{-1}\right)$ of the two substances.

\begin{tabular}{lcc}
\hline Absorption peak & Chitosan & Manufactured chitosan \\
\hline$-\mathrm{O}-\mathrm{H}$ & 3423,41 & 3420,43 \\
$-\mathrm{C}-\mathrm{H}$ & 2880,61 & 2920,43 \\
$-\mathrm{NH}_{2}$ & 1652,21 & 1553,40 \\
$-\mathrm{P}=\mathrm{O}$ & & 1247,57 \\
\hline
\end{tabular}
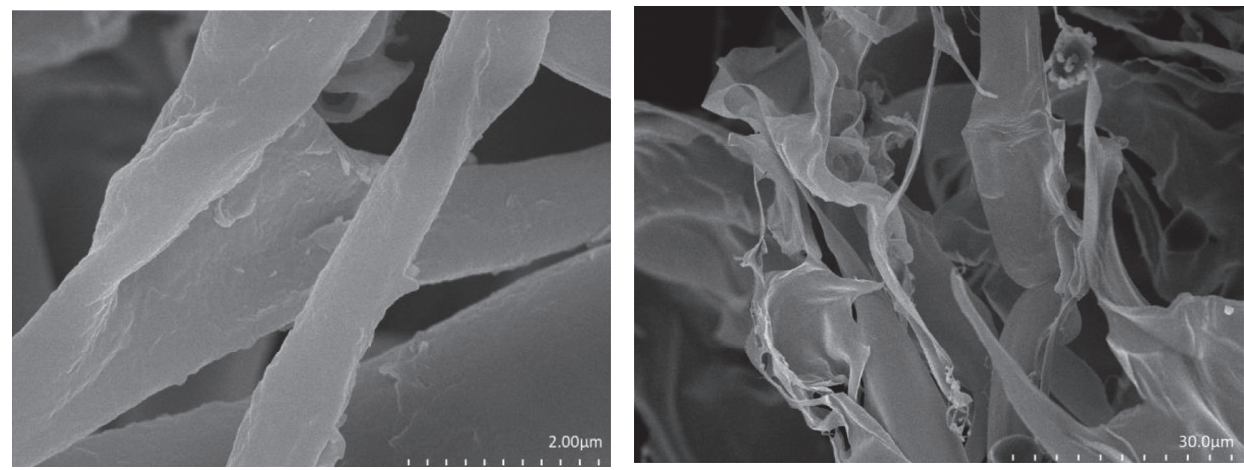

FIGURE 9: FE-SEM of original chitosan used for research.
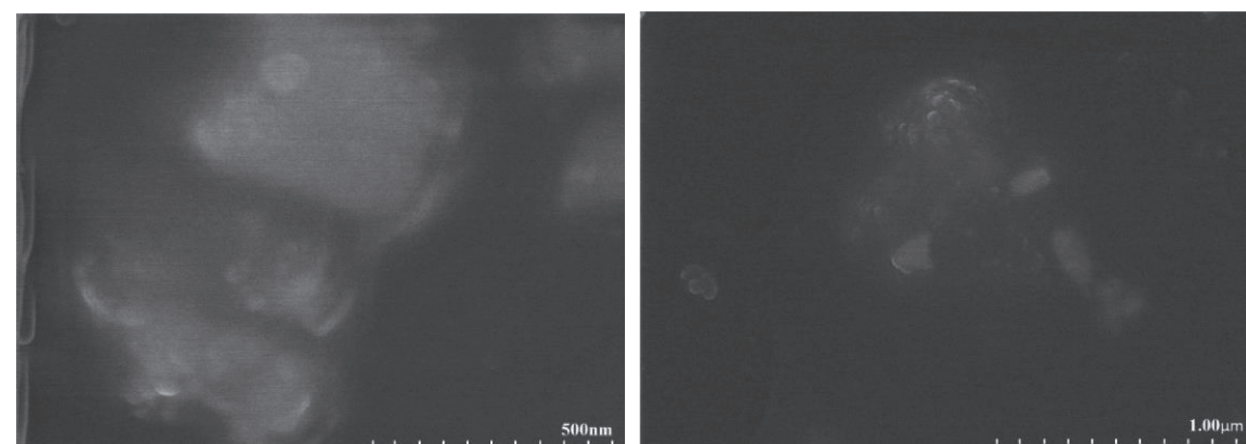

(a)
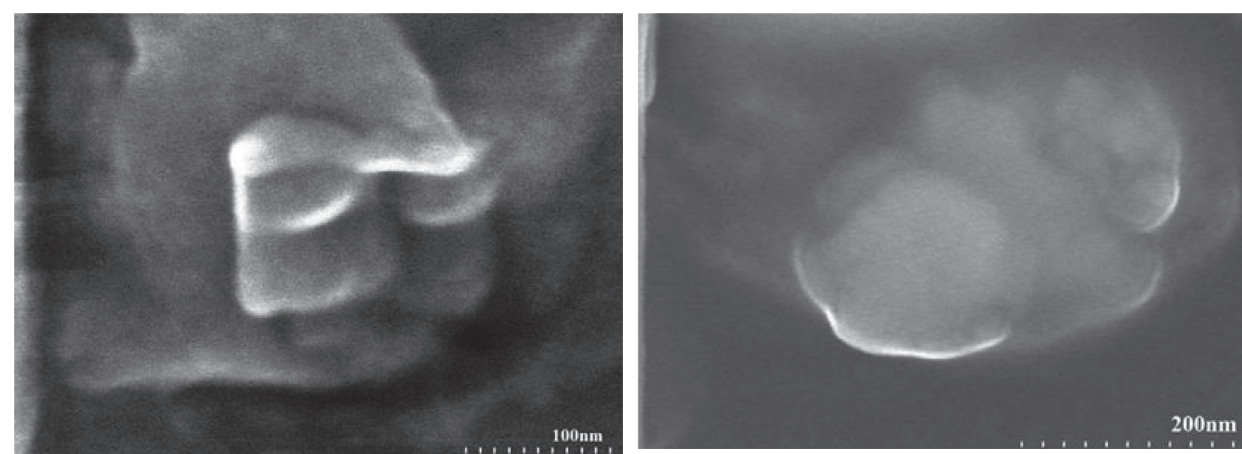

(b)
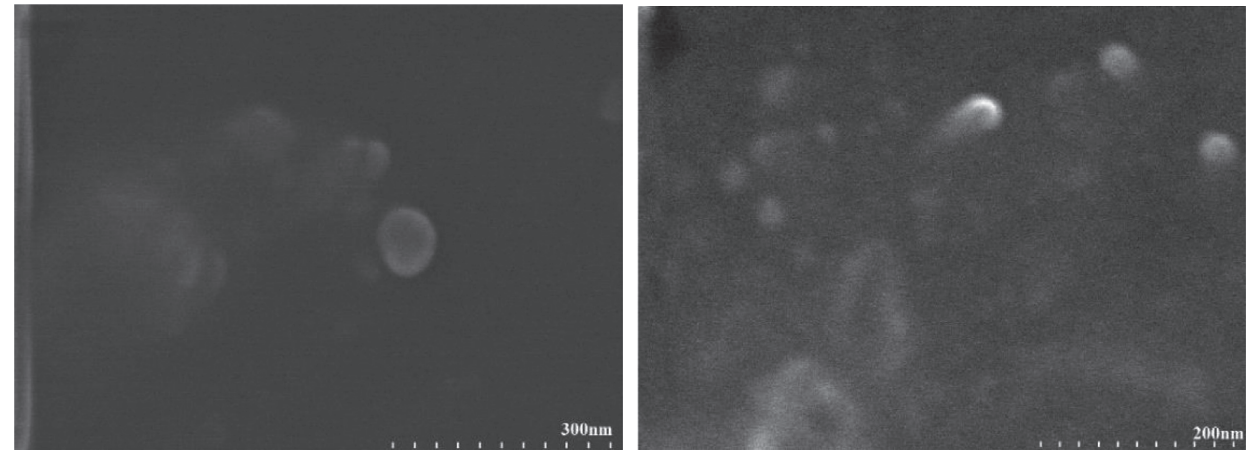

(c)

Figure 10: FE-SEM of chitosan/TPP at the ratios: (a) chitosan/TPP =8/2; (b) chitosan/TPP =4/2; (c) chitosan/TPP =6/3. 


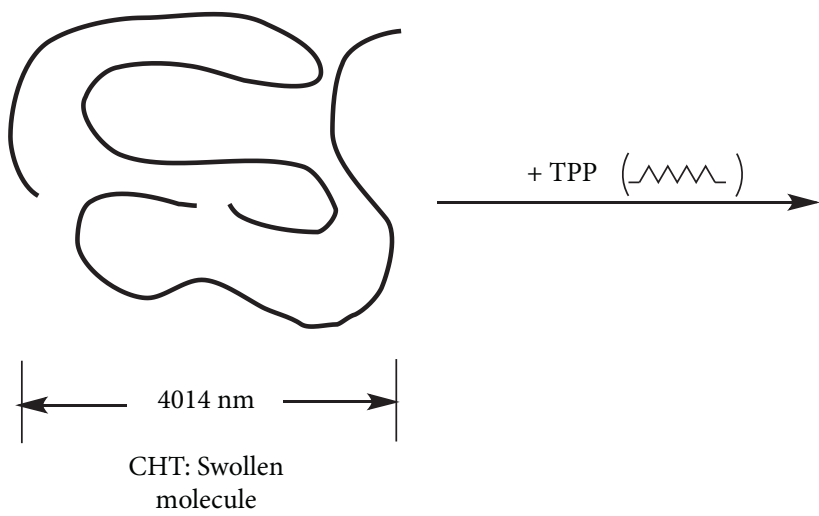

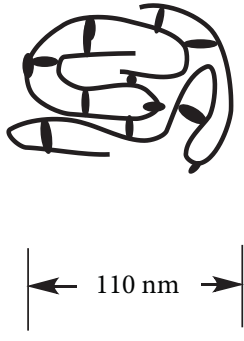

CHT-TPP nano matrix

Figure 11: Cross-linking ions of chitosan [3].

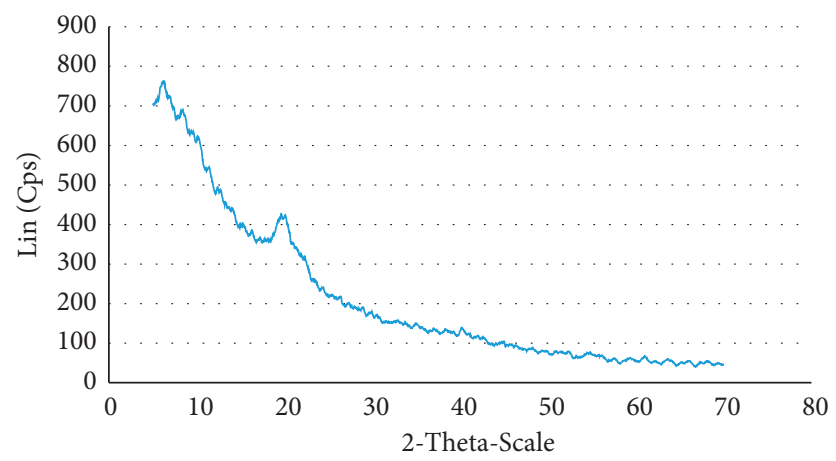

FIgURE 12: XRD diagram of the original chitosan.

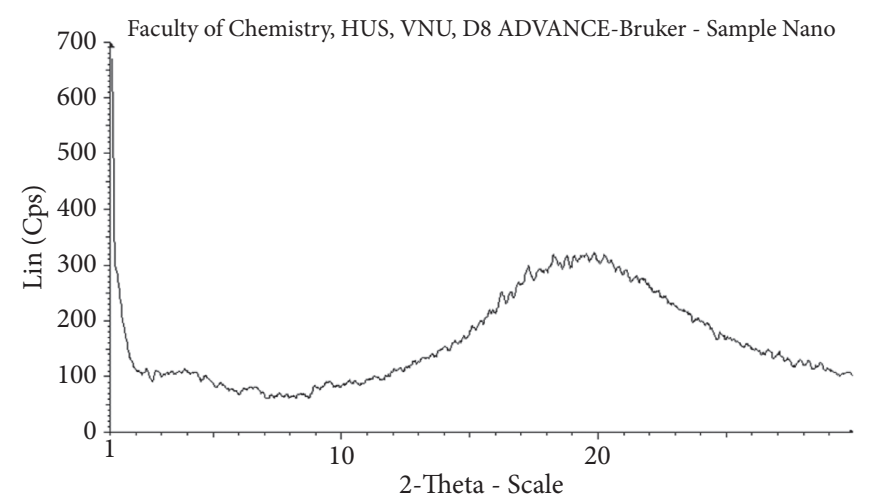

FIGURE 13: XRD diagram of chitosan/TPP manufactured at 6/3.
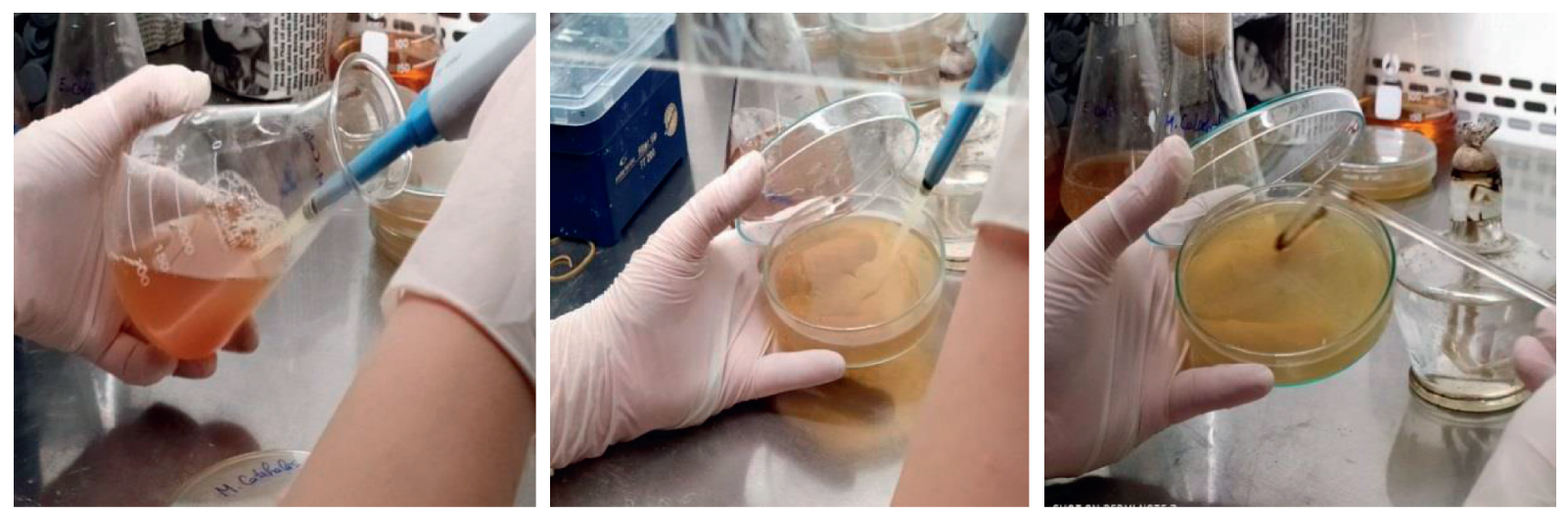

Figure 14: Steps to apply bacteria to agar plate. 

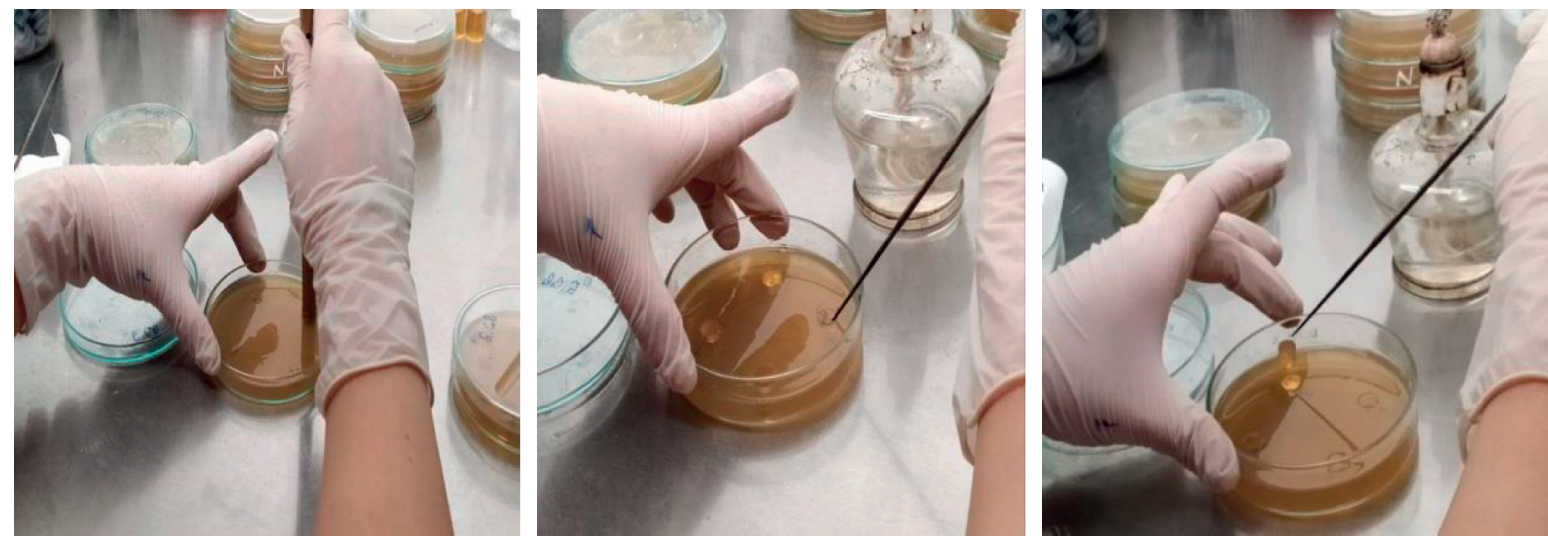

FIgURE 15: Steps to drill jelly wells.
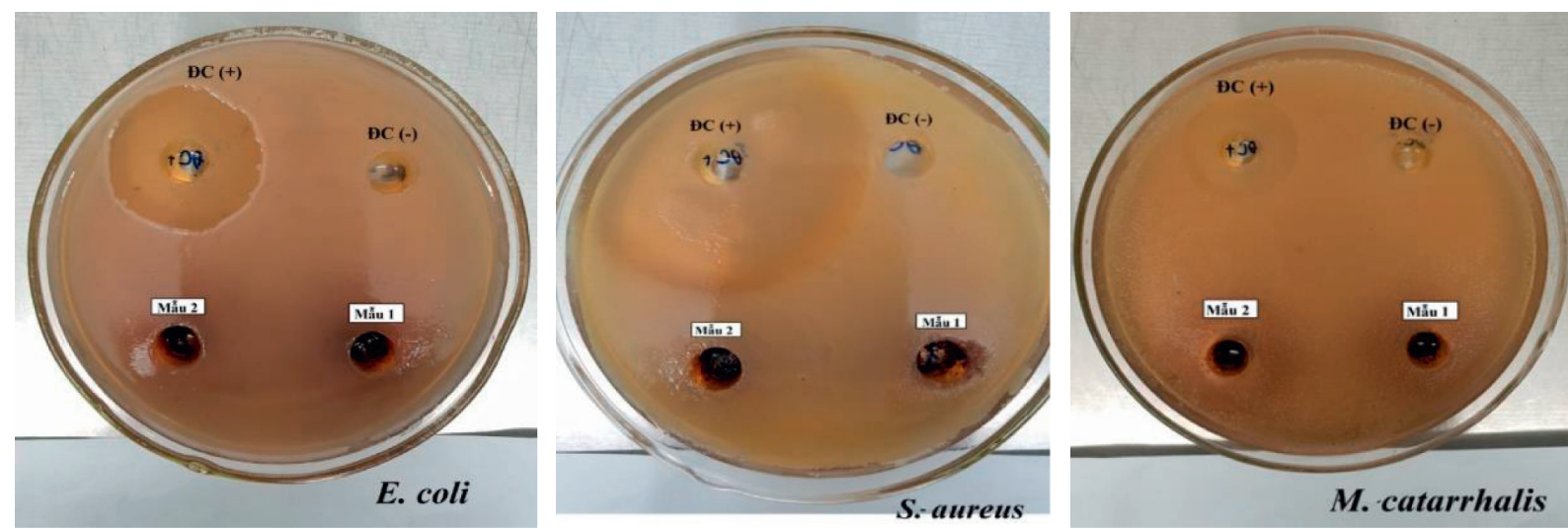

FIGURE 16: Antimicrobial test results: M01: nasal spray under study; M02: chitosan. First, check the reference results; the reference wells do not have problems to read the test results of the samples.
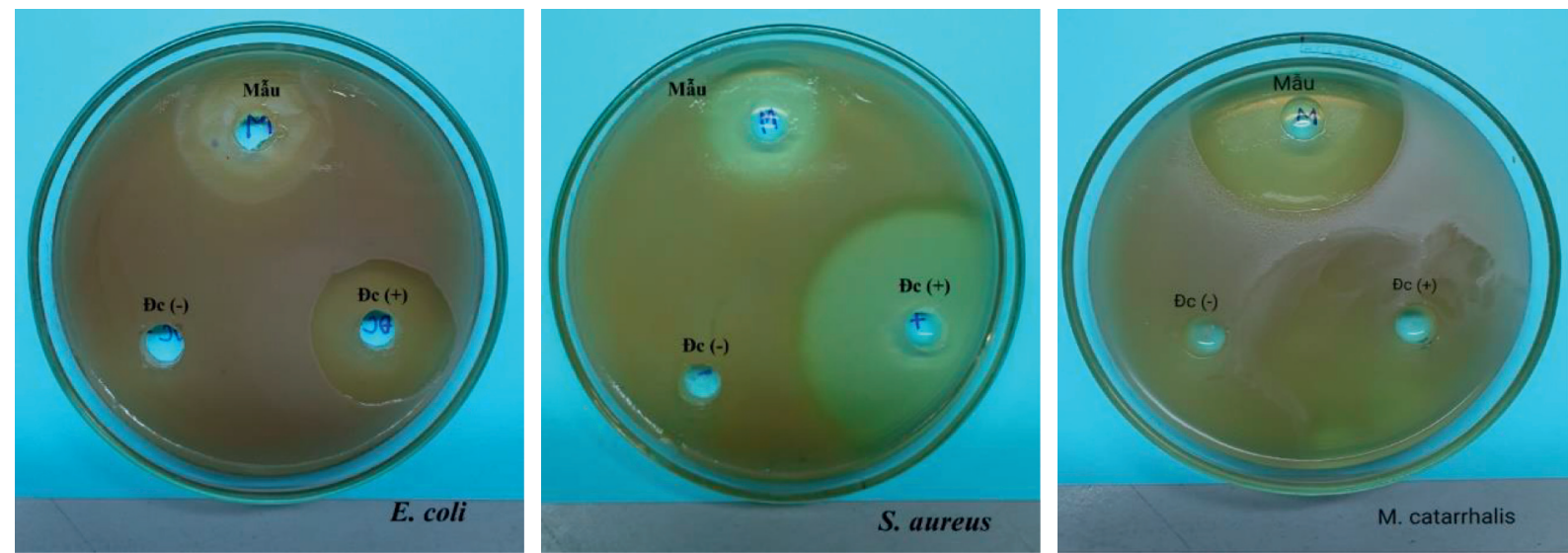

Figure 17: Result of testing antibacterial ability of chitosan suspension and nasal spray ( $5 \mathrm{ml}$ nasal spray and $5 \mathrm{ml}$ of chitosan suspension prepared at a concentration of $5 \mathrm{mg} / \mathrm{ml}$ ).

sample has antibacterial ability for all three strains of the tested bacteria: E. coli, M. catarrhalis, and S. aureus.

After three tests with the sample, the average diameter of the zone of inhibition of the sample is stated in the test results.

The sample 02 shows the test results of the drug and chitosan solution. The sample 03 is another reference sample. Comparing the zone of inhibition radius of sample
02 and sample 03 , we found that the zone of inhibition radius for strains of E. coli and M. catarrhalis decreased; only the zone of inhibition radius for $S$. aureus strain increased slightly from $8.2 \mathrm{~mm}$ to $9.3 \mathrm{~mm}$.

Chitosan nanoparticles (CNPs) confer higher antibacterial activity against a variety of fungi Gram-positive and Gramnegative bacteria when compared with chitosan. This suggests that CNPs have the potential to act as an antibacterial agent 
against pathogens. The main proposed antibacterial activity of CS is electrostatic interaction that alters membrane permeability. It then binds to DNA and disrupts DNA replication, leading to bacterial cell death [14]. Especially with low molecular weight, Chitosan can enter cells and bind to DNA, inhibiting replication [15]. The physiological activity of bacteria is disrupted and leads to bacterial cell death due to the accumulation of electronegative particles by chitosan in the cell [16].

\section{Conclusions}

(i) From the above research results, it can be seen that the zone of inhibition appears clearly for all three bacterial strains, showing that the manufactured chitosan/nasal spray Ageratum conyzoides L. sample has the antibacterial ability for the three test strains of bacteria: E. coli, M. catarrhalis, S. aureus.

(ii) The analysis results show that when adding a smallsized chitosan suspension to the nasal spray, Agerhinin initially did not have antibacterial properties, thereby confirming that the antibacterial properties of chitosan fabricated in combination with nasal sprays are effective and that chitosan with low molecular weight and small size from the original chitosan is successful and of scientific significance.

(iii) However, the study results show that when the particle concentration is low, due to the effect of the viscosity of the solution is insignificant, the drug is easily adsorbed on the particle surface, leading to increased chitosan adsorption efficiency when increasing the concentration of particles. When the particle concentration is high in the experimental conditions with strong vibration speed, the number of effective collisions between particles increases so that the particles stick together; the adsorption capacity of chitosan is reduced.

(iv) Chitosan with low molecular weight and small size has been confirmed to be antibacterial in many studies; however, this work shows that this ability depends on many factors such as molecular weight and chitosan concentration, and especially when chitosan is combined with the drug, more research is needed on the possible interaction between chitosan and the drug solution. These will be the team's next proposed studies.

\section{Data Availability}

The data used to support the findings of this study are available from the corresponding author upon request.

\section{Conflicts of Interest}

The authors declare that they have no conflicts of interest.

\section{Acknowledgments}

The authors wish to thank the Faculty of Chemical Technology, Hanoi University of Industry (HaUI), for funding this work.

\section{References}

[1] K.-S. Huang, Y.-R. Sheu, and I.-C. Chao, "Preparation and properties of nanochitosan," Polymer-Plastics Technology \& Engineering, vol. 48, no. 12, pp. 1239-1243, 2009.

[2] V. Kamat, I. Marathe, V. Ghormade, D. Bodas, and K. Paknikar, "Synthesis of monodisperse chitosan nanoparticles and in situ drug loading using active microreactor," ACS Applied Materials \& Interfaces, vol. 7, no. 41, pp. 22839-22847, 2015.

[3] D. P. Chattopadhyay and M. S. Inamdar, "Studies on synthesis, characterization and viscosity behaviour of nano chitosan," Esearch Journal of Engineering Sciences, vol. 1, no. 4, pp. 9-15, 2012.

[4] B. Pant, M. Park, G. P. Kim, H.-Y. Kim, and S.-J. Park, "Electrospun salicylic acid/polyurethane composite nanofibers for biomedical applications," International Journal of Polymeric Materials and Polymeric Biomaterials, vol. 67, no. 12, pp. 739-744, 2017.

[5] B. Pant, M. Park, and S.-J. Park, "One-step synthesis of silver nanoparticles embedded polyurethane nano-fiber/net structured membrane as an effective antibacterial medium," Polymers, vol. 2019, no. 1185, pp. 1-11, 2019.

[6] M. Ali and M. Hashemi, "Effect of chitosan molecular weight as micro and nanoparticles on antibacterial activity against some soft rot pathogenic bacteria," Lebensmittel-Wissenschaft und -Technologie- Food Science and Technology, vol. 71, pp. 347-355, 2016.

[7] K. A. M. O'Callaghan and J. P. Kerry, "Preparation of low- and medium-molecular weight chitosan nanoparticles and their antimicrobial evaluation against a panel of microorganisms, including cheese-derived cultures," Food Control, vol. 69, pp. 256-261, 2016.

[8] C. Gonçalves, N. Ferreira, and L. Lourenço, "Production of low molecular weight chitosan and chitooligosaccharides (COS): a review," Polymers, vol. 13, no. 2466, pp. 1-23, 2021.

[9] N. S. Chatterjee, P. K. Dara, S. Perumcherry Raman et al., "Nanoencapsulation in low-molecular-weight chitosan improves in vivo antioxidant potential of black carrot anthocyanin," Journal of the Science of Food and Agriculture, vol. 101, no. 12, pp. 5264-5271, 2021.

[10] V. O. Fasiku, C. A. Omolo, N. Devnarain et al., "Chitosan-based hydrogel for the dual delivery of antimicrobial agents against bacterial methicillin-resistant Staphylococcus aureus biofilm-infected wounds," ACS Omega, vol. 6, no. 34, pp. 21994-22010, 2021.

[11] M. Safarzadeh and S. Taheri, "Highly monodisperse chitosan nanoparticles prepared by a combined triple method for potential use as drug carriers," International journal of nanomaterials, nanotechnology and Nanomedicine, vol. 3, no. 1, pp. 1-6, 2017.

[12] S Sreekumar, F. M. Goycoolea, B. M. Moerschbacher, and G. R. Rivera-Rodriguez, "Parameters influencing the size of chitosan-TPP nano- and microparticles," Scientific Reports, vol. 8, no. 4695, pp. 4695-4711, 2018.

[13] Li. Qi, Z.. Xu, X. Jiang, C. Hu, and X. Zou, "Preparation and antibacterial activity of chitosan nanoparticles," Carbohydrate Research, vol. 339, p. 2, 2004.

[14] M. Chandrasekaran, "Antibacterial activity of chitosan nanoparticles: a review," Processes, vol. 8, no. 1173, pp. 1-21, 2020.

[15] K. Birsoy, T. Wang, W. W. Chen, E. Freinkman, M. AbuRemaileh, and D. M. Sabatini, "An essential role of the mitochondrial electron transport chain in cell proliferation is to enable aspartate synthesis," Cell, vol. 162, no. 3, pp. 540-551, 2015.

[16] K. Eltahlawy, M. Elbendary, A. Elhendawy, and S. Hudson, "The antimicrobial activity of cotton fabrics treated with different crosslinking agents and chitosan," Carbohydrate Polymers, vol. 60, no. 4, pp. 421-430, 2005. 